\title{
Effect of Supplementation Yeast Fermentation Products on Growth Performance and Intestinal Health of Weaned Piglets Challenged With Salmonella Typhimurium
}

\author{
Yongsen Zhao \\ Nanjing Agricultural University College of Veterinary Medicine \\ Danping Wang \\ Nanjing Agricultural University College of Veterinary Medicine \\ Meng Jiang \\ Nanjing Agricultural University College of Veterinary Medicine \\ Jinglong Chen \\ Nanjing Agricultural University College of Veterinary Medicine \\ Xiaojing Yang ( $\sim$ yangxj@njau.edu.cn ) \\ Nanjing Agricultural University College of Veterinary Medicine
}

\section{Research}

Keywords: yeast fermentation product, growth performance, intestinal inflammation, apoptosis, piglets

Posted Date: January 15th, 2021

DOI: https://doi.org/10.21203/rs.3.rs-143524/v1

License: (c) (i) This work is licensed under a Creative Commons Attribution 4.0 International License.

Read Full License 


\section{Abstract}

Background: This study investigated the effects of piglets with dietary supplementation yeast fermentation product (YFP) on growth performance, immune status and intestinal inflammation after a Salmonella typhimurium challenge. Twenty-four weaned piglets were assigned to four treatments including: non-challenge control (Con); Salmonella typhimurium-challenged control (ST); ST + $0.2 \%$ YFP $(0.2 \%$ YFP); and ST $+0.4 \%$ YFP $(0.4 \%$ YFP $)$. All piglets were challenged twice with Salmonella typhimurium. All of them were killed at $7^{\text {th }}$ day after the second challenge to obtain plasma and intestine for analysis.

Results: $0.4 \%$ YFP increased average daily gain (ADG) and duodenal villus height and villus height/ crypt depth $(P<0.05)$ and decreased feed-gain ratio $(P<0.05)$ after Salmonella typhimurium challenge compared with ST group. The $0.4 \%$ YFP decreased the elevating concentrations IL- $1 \mathrm{~b}$ and IL-6 $(P<0.01)$ induced by Salmonella typhimurium and increased the concentration of IL-10 $(P<0.05)$ in plasma compared with ST group. Furthermore, YFP influenced the apoptosis related mRNA levels of Bax, Bcl-2 and caspase $3(P<0.05)$ and increased intestinal occludin protein expression $(P<0.05)$. 0.4\% YFP downregulated the mRNA expressions of TLR4, MyD88, IRAK1, TRAF6 and NFkB $(P<0.05)$ and decreased the mRNA expression of IL-6, TNFa $(P<0.05)$ and increased the IL-10 $(P<0.01)$ in duodenum compared with ST group. In addition, $0.4 \%$ YFP decreased the phosphorylation levels of p38 MAPK and ERK $1 / 2(P<$ $0.01)$.

Conclusions: Dietary $0.4 \%$ yeast fermentation product supplementation had positive effects on growth performance and intestinal barrier function and reduced intestinal inflammation of weaned piglets challenged with Salmonella typhimurium.

\section{Background}

Due to the risk of emergence of resistant bacteria and resistance in humans, China has already banned the use of antibiotics in feed from 2020, though amounts of studies have reported that the use of antibiotics can improve growth performance and protect intestinal infections of weaned piglets [1-3]. Therefore, increasing attention has focused on searching for alternatives to antibiotics.

The effects of dietary supplementation with yeast fermentation products on the improvement of intestinal health could therefore be of great importance in pigs. Yeast fermentation products contain amounts of bioactive compounds including nucleotides, nutritional metabolites and cell wall polysaccharides (specifically $\beta$-glucan and mannan). Several studies have investigated the impact of yeast cell wall components on immune function of weaning piglets [4-7]. It has been reported that yeast $\beta$-glucans alleviated the elevated of pro-inflammation cytokines and enhanced the production of antiinflammatory cytokines in LPS or Escherichia coli challenged piglets [8, 9]. Dietary supplementation yeast-derived mannans could improve piglets' weight gain and enhanced immune function [10]. Since 
yeast fermentation products are beneficial to the growth of piglets, it has been considered to be a potential in-feed antibiotic alternative.

However, how the influence of yeast fermentation products on intestinal health on the condition of Salmonella typhimurium challenge is unclear and the underlying mechanism is not documented. Therefore, this study focused on indicating how yeast fermentation products improved growth performance and intestinal health for piglets challenged with Salmonella typhimurium. Our study will provide potentiality to find alternatives to antibiotics.

\section{Materials And Methods}

\subsection{Animals and experimental design}

A total of 24 weaned male piglets (Duroc - Yorkshire - Landrace) with an initial average body weight of were housed at Laboratory Animal Center of Nanjing Agricultural University. The piglets used did not receive Salmonella typhimurium vaccines, antibiotic injections or antibiotics in feed. All piglets used in this study were susceptible to Salmonella typhimurium. Piglets were housed in pens (pen size: $1.2 \mathrm{~m}$. $1 \mathrm{~m}$ ) in an environmental controlled nursery building. Each pen was equipped with a feeder, a nipple drinker and plastic-covered expanded metal floors. The room temperature was maintained at $25 \pm 1{ }^{\circ} \mathrm{C}$ throughout the study. The piglets had free access to feed and water. The yeast fermentation products are provided by Cargill Company ( XPC, Diamond V, Cedar Rapids, IA, USA).

The experiment lasted for 5 weeks, including 21 days before and 14 days after the first Salmonella typhimurium challenge. All piglets were randomly allotted to four dietary treatments $(n=6)$ based on the initial body weight included: (1) Negative control (Con): control diet, without salmonella typhimurium challenge; (2) Positive control (ST): control diet, with salmonella typhimurium challenge; (3) $0.2 \%$ YFP: control diet plus $2 \mathrm{~g} / \mathrm{kg}$ feed, with salmonella typhimurium challenge; (4) $0.4 \%$ YFP: control diet plus $4 \mathrm{~g} / \mathrm{kg}$ feed, with salmonella typhimurium challenge (Table 1 ). The experimental diets were fed to pigs through the study duration. After 21 days experimental diets feeding, the piglets of the last three groups

orally inoculated with $5 \mathrm{~mL}$ suspension containing $10^{9} \mathrm{CFU}$ of Salmonella typhimurium. A week later, the second challenge was performed the same as the first. Body weight of per piglet was recorded at days 0 , $7,14,21$ and 35 and feed consumption per pen was recorded every day of the experiment to calculate the average daily gain (ADG), average daily feed intake (ADFI) and feed efficiency (F:G) from d 1 to 21 (prechallenge) and $d 21$ to 35 (post-challenge). 
Table 1

Ingredient composition of experimental diets (\%, as-fed basis)

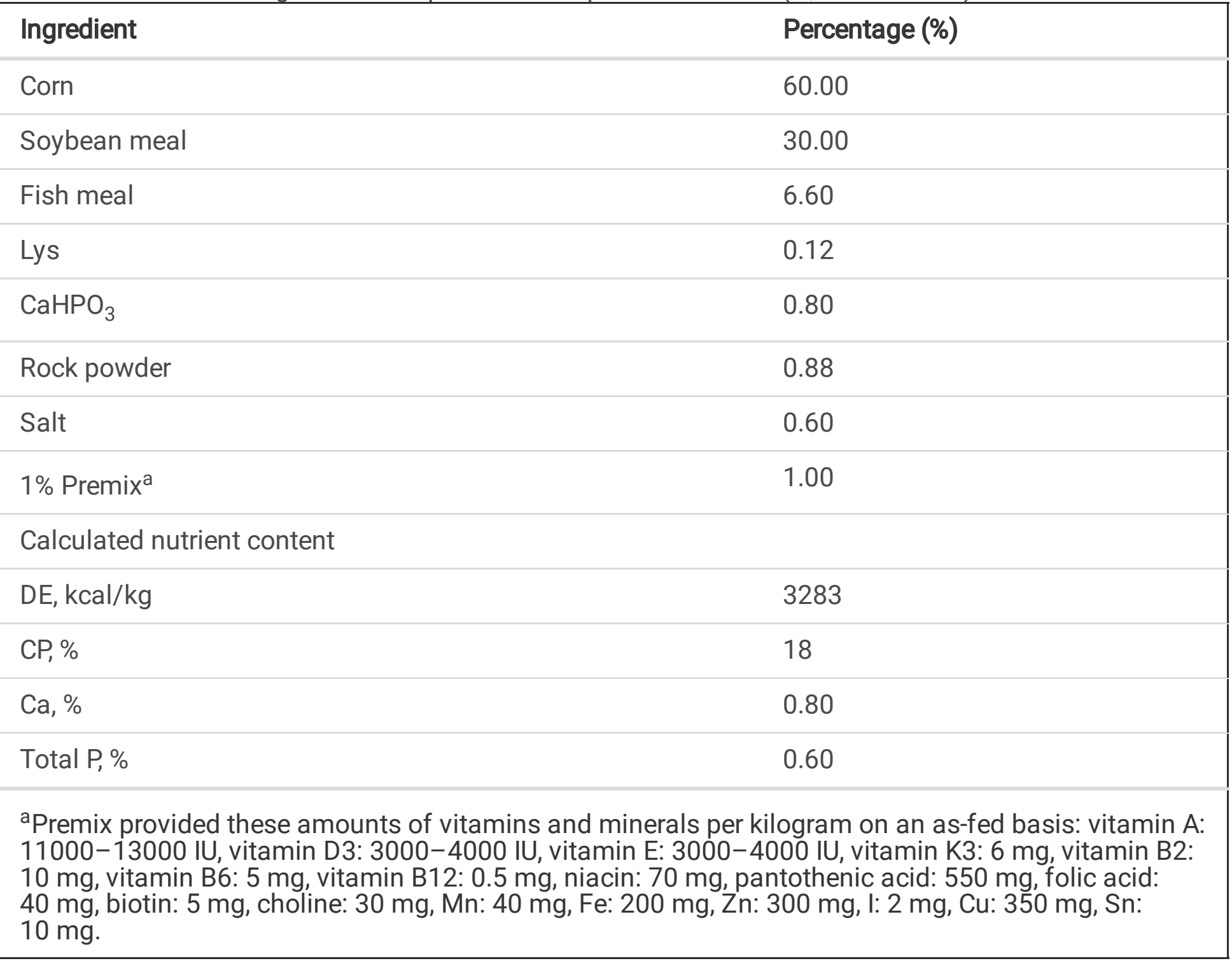

\subsection{Sample collections}

All the piglets were sacrificed by exsanguination at the end of the study. Blood samples were collected into heparinized tubes. Plasma was separated by centrifugation at $3000 \mathrm{rpm} / \mathrm{min}$ for $15 \mathrm{~min}$ and stored at $-20^{\circ} \mathrm{C}$ until analysis. Once dead, the abdomen was immediately opened, and the intestinal tract was excised. Duodenum segments (approximately $8 \mathrm{~cm}$ in length) were opened longitudinally and gently flushed with sterile saline to remove the contents. Duodenal mucosa samples were collected by lightly scraping with sterile glass slides on rice, immediately frozen in liquid nitrogen and stored at $-80{ }^{\circ} \mathrm{C}$ for analysis. 3-cm sections from the duodenum (to about $10 \mathrm{~cm}$ distal to the pylorus) were collected and fixed in $4 \%$ paraformaldehyde for intestinal morphology analysis.

\subsection{Intestinal morphology analysis}

Duodenal segments were removed from the stationary solution and then were embedded in paraffin. Sections $(3 \mu \mathrm{m})$ were stained with hematoxylin and eosin $(\mathrm{HE})$ to investigate architecture of the 
duodenum. Stained slices were scanned with the Pannoramic SCAN II and images were captured with 3DHISTECH software (3DHISTECH Ltd. Budapest, Hungary).

\subsection{Plasma inflammatory cytokine concentration analysis}

Plasma IL-1 $\beta$, IL- 6 and IL-10 concentrations were measured using the ELISA kits suitable for porcine IL-1 $\beta$, IL-6 and IL-10 (Jiangsu MEIMIAN Industrial Co. LTD, China), respectively, according to the manufacture's protocol. Plasma concentrations of IL-1 $1 \beta, \mathrm{IL}-6$ and IL-10 were calculated from the standard curve and expressed as $\mathrm{ng} / \mathrm{L}$.

\subsection{Total RNA isolation and real-time PCR}

Total RNA was isolated from $30 \mathrm{mg}$ frozen duodenal mucosa with $1 \mathrm{~mL}$ TRIzol (Sangon Biotech, Shanghai, China) and reverse-transcribed according to the manufacturer's protocol (Vazyme Biotech, Nanjing, China). Diluted cDNA ( $2 \mu \mathrm{L}, 1: 25)$ was used as template for real-time PCR that was performed on a real-time PCR system (Mx3000P, Stratagene, USA). Moreover, GAPDH was chosen as a reference gene to normalize the mRNA abundance of target genes. All primers were synthesized by and listed in Table 2 . The $2^{-\triangle \Delta C T}$ method was used to analyze real-time PCR data.

Table 2

The primer sequences for RT-PCR.

\begin{tabular}{|lll|}
\hline Target genes & Primer sequences (5' $^{\prime}$ to $\left.\mathbf{3}^{\prime}\right)$ & \\
\hline IL-1 $\beta$ & F: ACATGCTGAAGGCTCTCCAC & R: CAGGGTGGGCGTGTTATCTT \\
\hline IL-6 & F: GCAGTCACAGAACGAGTGGA & R: CTCAGGCTGAACTGCAGGAA \\
IL-10 & F: CGGCCCAGTGAAGAGTTTCT & R: TGCCTTCGGCATTACGTCTT \\
TNFa & F: GCCCTTCCACCAACGTTTTC & R: CAAGGGCTCTTGATGGCAGA \\
TLR4 & F: CGTGCAGGTGGTTCCTAACA & R: AAAGGCTCCCAGGGCTAAAC \\
MyD88 & F: CCATTCGAGATGACCCCCTG & R: TAGCAATGGACCAGACGCAG \\
NFKB & F: CGGGGACTACGACCTGAATG & R: CTTTCTGCACCTTGTCGCAC \\
IRAK1 & F: GCAGTTGTCACGGTTTCGTC & R: GAAGTCTCCCAGTTTGGGCA \\
TRAF6 & F: CCAGAGACCCACAATCCCAC & R: ACCCTCCCTCCGAAGACTAC \\
Bax & F: GACAGGGGCCCTTTTGCTTC & R: CCGCCACTCGGAAAAAGACT \\
Bcl-2 & F: GAACTGGGGGAGGATTGTGG & R: GCCGGTTCAGGTACTCAGTC \\
Caspase3 & F: GTGGGACTGAAGATGACA & R: ACCCGAGTAAGAATGTG \\
\hline
\end{tabular}

\subsection{Western blot analysis}

Total protein was extracted from $50 \mathrm{mg}$ frozen samples as previously described [11]. Protein concentration was measured with Pierce BCA Protein Assay kit (No. 23225, Thermo Scientific) according 
to manufacturer's instruction. Western blot analysis of Occludin (DF7504, Affinity, diluted 1:1000), HSP70 (AF5466, Affinity, diluted 1:1000), Bax (BS6420, Bioworld, diluted 1:1000), TLR4 (SC293072, Santa Cruz, diluted 1:200), p38 (AF6456, Affinity, diluted 1:1000), p-p38 (AF4001, Affinity, diluted 1:1000), ERK1/2 (AF0155, Affinity, diluted 1:1000), p-ERK1/2 (AF1015, Affinity, diluted 1:1000) were carried out. The $\beta$-actin (AC026, ABclonal, diluted 1;50000) was used as internal control.

\subsection{Statistical analysis}

All data were checked for normality using exploratory analysis. Data were analyzed using SPSS 21.0 for Windows (SPSS Inc., Chicago, IL, USA). All data were analyzed using One-Way ANOVA with diet (YFP) as a fixed factor. The piglets were recognized as a statistical unit. The values were presented as the means \pm SEM with significance at $P<0.05$.

\section{Results}

\subsection{Growth performance}

Salmonella typhimurium administration tended $(P=0.09)$ to decrease the average daily gain (ADG) compared with Con group, yet $0.4 \%$ YFP supplement was significantly $(P<0.05)$ increased compared with ST group. The ratio of feed and gain $(F / G)$ was observed to significantly increase with Salmonella typhimurium challenged compared with control group and it reversed by $0.4 \%$ YFP $(P<0.05)$. No significant changes were observed in ADG or F/G between control and YFP supplement groups before Salmonella typhimurium treatment (Table 3). 
Table 3

Growth performance

\begin{tabular}{|c|c|c|c|c|c|c|c|}
\hline \multirow[t]{2}{*}{ Items } & \multirow[t]{2}{*}{ Con } & \multirow[t]{2}{*}{ ST } & \multirow[t]{2}{*}{$0.2 \%$ YFP } & \multirow[t]{2}{*}{$0.4 \%$ YFP } & \multicolumn{3}{|c|}{$P$ Value } \\
\hline & & & & & P 1 & P 2 & P 3 \\
\hline \multicolumn{8}{|c|}{ Pre-challenge } \\
\hline ADG $(\mathrm{Kg})$ & $0.49 \pm 0.02$ & $0.45 \pm 0.03$ & $0.45 \pm 0.04$ & $0.48 \pm 0.02$ & 0.384 & 0.975 & 0.498 \\
\hline ADFI (Kg) & $0.73 \pm 0.02$ & $0.80 \pm 0.02$ & $0.74 \pm 0.01$ & $0.76 \pm 0.03$ & 0.053 & 0.104 & 0.219 \\
\hline$F / G$ & $1.51 \pm 0.07$ & $1.72 \pm 0.09$ & $1.69 \pm 0.15$ & $1.68 \pm 0.06$ & 0.131 & 0.808 & 0.738 \\
\hline \multicolumn{8}{|c|}{ Post-challenge } \\
\hline ADG (Kg) & $0.66 \pm 0.03$ & $0.51 \pm 0.04$ & $0.52 \pm 0.09$ & $0.69 \pm 0.04$ & 0.094 & 0.920 & 0.048 \\
\hline ADFI (Kg) & $1.13 \pm 0.02$ & $1.05 \pm 0.06$ & $1.04 \pm 0.12$ & $1.15 \pm 0.04$ & 0.531 & 0.886 & 0.358 \\
\hline$F / G$ & $1.76 \pm 0.09$ & $2.09 \pm 0.03$ & $2.00 \pm 0.13$ & $1.81 \pm 0.09$ & 0.023 & 0.533 & 0.043 \\
\hline \multicolumn{8}{|c|}{ P 1: ST vs Con } \\
\hline \multicolumn{8}{|c|}{ P $2: 0.2 \%$ YFP vs ST } \\
\hline \multicolumn{8}{|c|}{ P 3: $0.4 \%$ YFP vs ST } \\
\hline The values & sented as $\mathrm{m}$ & an \pm SEM & & & & & \\
\hline
\end{tabular}

Salmonella typhimurium-challenged had higher concentrations of plasma IL-1 $(P<0.01)$ and IL-6 $(P=$ $0.001)$ and lower concentration of plasma IL-10 $(P<0.05)$ than the Con piglets. Compared with ST piglets, the concentrations of plasma IL- $1 \beta(P<0.01)$ and IL- $6(P<0.01)$ was lower in $0.4 \%$ YFP piglets, meanwhile the concentration of IL-10 was higher $(P<0.05)$ in plasma (Fig. 1).

\subsection{Intestinal morphology}

Compared with the Con, the duodenum villus height and villus height/crypt depth were significantly lower $(P<0.01)$ in the ST group; however, the crypt depth was not affected by Salmonella typhimurium. The villus height and villus height/crypt depth were reversed to the control levels by $0.2 \%$ or $0.4 \%$ YFP exposure (Fig. 2).

\subsection{Intestinal occludin and heat shock protein 70}

Salmonella typhimurium challenge affected occludin $(P<0.05)$ (Fig. 3A) and HSP70 $(P<0.05)$ (Fig. 3B) protein expression in duodenum compared with Con group. Piglets fed $0.4 \%$ YFP had higher occludin $(P<$ 0.05) protein expression, while YFP did not alleviate the increased HSP70 protein expression. 


\subsection{Intestinal apoptosis-related gene abundances and protein expression}

The relative mRNA abundances of Bcl2-associated X protein (Bax) $(P<0.01)$ and cysteinyl aspartate specific protease 3 (caspase 3$)(P<0.001)$ were up-regulated by Salmonella typhimurium treatments; however, they were down-regulated by $0.4 \%$ YFP addition. B-cell lymphoma-2 $(\mathrm{Bcl}-2)(P<0.05) \mathrm{mRNA}$ abundance was down-regulated by Salmonella typhimurium treatments, $0.4 \%$ YFP addition up-regulated the mRNA abundance (Fig. 4A). Furthermore, Bax protein expression in the ST groups was significantly higher $(P<0.05)$ than the control group, but $0.2 \%$ or $0.4 \%$ YFP addition retrieved it (Fig. 4B). These results deduced that YFP could alleviate the intestine mucous apoptosis induced by Salmonella typhimurium.

\subsection{Intestinal TLR4 signaling pathway expression}

Piglets challenged with Salmonella typhimurium had higher mRNA abundance of TLR4 $(P<0.01)$, IL-1 receptor-associated kinase 1 (IRAK 1$)(P<0.01)$, NFKB $(P<0.05)$ and TNF-a receptor-associated factor 6 (TRAF 6) $(P<0.01)$ compared with the Con. 0.4\% YFP supplementation significantly decreased mRNA abundance of TLR4, MyD88, IRAK1, NFKB and TRAF6 $(P<0.05)$ (Fig. 5A), the same as TLR4 protein expression $(P<0.05)$ (Fig. 5B).

\subsection{Intestinal inflammation cytokines mRNA expression}

Salmonella typhimurium-challenged significantly increased the mRNA expression of IL-1 $\beta$, IL- 6 and TNFa $(P<0.05)$ and significantly decreased IL-10 $(P<0.01)$ mRNA expression compared with the Con and $0.4 \%$ YFP addition could decrease IL- 6 and TNFa mRNA expression and increase IL-10 mRNA expression. (Fig. 6).

\subsection{Intestinal MAPK signaling pathway expression}

Compared with the Con piglets, the phosphorylation levels of ERK (Fig. 7A) and p38 (Fig. 7B) were significantly increased $(P<0.01)$ in the Salmonella typhimurium-challenged piglets. Meanwhile, $0.2 \%$ and $0.4 \%$ YFP all significantly inhibited the activation of the key proteins in the MAPK pathway compared with ST group.

\section{Discussion}

Yeast fermentation product is used widely in the livestock industry. The potential mechanisms of action of yeast products that maintains an advantageous intestinal environment [12] by regulating microbial ecology and elevates intestinal immunity through preventing pathogenic bacteria from intestinal epithelial cells, consequently improving growth performance have been reported $[13,14]$. In this study, during the pre-challenge period, supplementation of $0.2 \%$ and $0.4 \%$ YFP had no significant effect on growth performance of piglets compared with the control. However, during the Salmonella typhimurium challenge period, $0.4 \%$ YFP significantly enhanced ADG compared with ST group. In general, the present study indicates that the relatively high dose of YFP offered protection against Salmonella typhimurium 
and that is beneficial to enhance growth performance of piglets during Salmonella typhimurium infection. The following discussion was focused on the $0.4 \%$ YFP effects after Salmonella typhimurium infection.

Intestinal morphology can be used as an indicator for intestinal health [15]. To response inflammation induced by pathogens, deeper crypts exhibited faster cellular turnover allowing renewal of villus as needed [16]. In the current study, dietary YFP supplementation improved intestinal histomorphology in the infectious piglets. Salmonella typhimurium challenge induced significantly deeper crypt and shorter villus in the duodenum, the same observations reported challenged with Salmonella typhimurium in piglets. In the challenged piglets, however, the dietary YFP supplementation remarkably increased villus height and decreased crypt depth, which contributed significantly to enhance V/C ratio, indicating the effective nutrient absorption capability and the decreased F/G.

Tight junctions among intestinal epithelial cells, which protect the body from intestinal pathogens, influence intestinal mucosal barrier function to a great extent [17]. Occludin, as the major integral proteins, form the continuous tight junction strands [18]. In the current study, YFP supplementation significantly increased occludin expression in duodenum, which is expected to improve intestinal mucosal barrier function. Our results demonstrated that YFP kept intestinal integrity and barrier function partially by elevating intestinal tight junction protein expression.

Currently, apoptosis is derived by the expression of Bax, Bcl-2 and caspase 3 [19]. In this study, the mRNA abundances of Bax and caspase 3 were significantly up-regulated, and Bax protein expression level was also increased in the ST group, demonstrating that ST induced intestinal epithelial cell apoptosis, but both mRNA abundances and Bax protein expression were down-regulated with supplementation YFP. Our findings showed that YFP might protect intestinal barrier function by inhibiting apoptosis pathway.

LPS induces inflammation in pigs by stimulating the production of cytokines such as IL-1, IL-6 and TNFa, which are considered as endogenous mediators of inflammation [20]. IL-10 is an important antiinflammatory cytokine which is required for protection in the regulation of intestine homeostasis during host defense [21, 22]. HSP70 also plays a significant role on intestinal inflammation response. The concentrations of plasma IL-1 $\beta$, IL- 6 and IL-10 were measured as indicators of systemic pro- and antiinflammation responses, respectively. In the present study, the increased plasma IL- $1 \beta$ and IL- 6 concentrations indicated successful establishment of the Salmonella typhimurium challenge model. And Salmonella typhimurium caused an increased of intestinal HSP70 protein expression. Piglets receiving YFP had less concentration of plasma IL-1 $\beta$ and IL- 6 and higher concentration of IL-10 than ST group, hence, implying that YFP lowered pro-inflammation of the immune system. The overproduction of proinflammatory cytokines is associated with anorexia, which may explain the observed reduction in ADG in Salmonella typhimurium-challenged piglets in our study $[23,24]$. Meanwhile, the evidence that lower mRNA expression of IL-1 $\beta$, IL-6 and TNFa, and higher IL-10 expression compared to the ST piglets in duodenum indicated that dietary supplementation with YFP contributed to beneficial immunoregulatory responses. 
Toll like receptor 4 (TLR4) is the first identified of TLRs family, which recognizes the LPS component of Gram negative bacteria on the cell surface [25]. The activation of TLR4 signaling pathway activates members of the MAPK family including p38 and ERK1/2, leading to numerous pro-inflammatory cytokine genes translation [26]. TLR4 and MAPK signaling are of great significance in intestinal inflammation [27, 28]. In the present study, ST challenge increased mRNA expression of TLR4 and its downstream signals IRAK1, TRAF6 and NFKB. YFP supplementation decreased TLR4, MyD88, IRAK1, TRAF6 and NFKB mRNA expression and TLR4 protein, p38 phosphorylation and ERK1/2 phosphorylation. Duan et al. [29] demonstrated that mannan oligosaccharide (a component of YFP) supplementation could enhance intestinal mucosal immune competence and suppress intestinal inflammation by decreasing the contents of pro-inflammatory cytokines. Sun et al. [30] demonstrated that saccharomyces cerevisiae polysaccharide (a component of YFP) could decrease the pro-inflammation mediators of IL- $1 \beta$ and IL- 6 at protein and mRNA levels by inhibiting MAPK activity in mice with DSS-induced colitis, which were consistent with our results.

\section{Conclusion}

Dietary yeast fermentation products supplementation could suppress Salmonella typhimurium hazards. And salmonella typhimurium-challenged piglets fed diets supplementation with $0.4 \%$ yeast fermentation products exhibited better growth performance possibly by alleviating intestinal inflammation and apoptosis to improve intestinal health.

\section{Declarations}

\section{Acknowledgments}

This study was supported by the National Natural Science Foundation of China (31772696 and 32072808), and the Priority Academic Program Development of Jiangsu Higher Education Institutions.

\section{Authors' contributions}

Yongsen Zhao contributed to all data analysis and drafting of the manuscript. Danping Wang and Meng Jiang were responsible for animal care, breeding and sampling. Jinglong Chen provided technical support. Xianjing Yang contributed to the experimental design, data interpretation and critical revision of the manuscript.

\section{Availability of data and materials}

The data analyzed in this study are available from the corresponding author on reasonable request.

\section{Ethics approval and consent to participate}

All procedures with animals were approved by the Animal Ethics Committee of Nanjing Agricultural University. The sampling procedures followed the "Guidelines on Ethical Treatment of Experimental 
Animals" (2006) No. 398 set by the Ministry of Science and Technology, China.

\section{Consent for publication}

Not applicable

\section{Conflict of interest}

The authors declare that they have no conflict of interest.

\section{References}

1. Vanrolleghem W, Tanghe S, Verstringe S, Bruggeman G, Papadopoulos D, Trevisi P, et al. Potential dietary feed additives with antibacterial effects and their impact on performance of weaned piglets: A meta-analysis. Veterinary Journal (London, England: 1997). 2019;249:24-32.

2. Walsh MC, Sholly DM, Hinson RB, Saddoris KL, Sutton AL, Radcliffe JS, et al. Effects of water and diet acidification with and without antibiotics on weanling pig growth and microbial shedding. Journal of Animal Science. 2007;85:1799-808.

3. Weber TE, Schinckel AP, Houseknecht KL, Richert BT. Evaluation of conjugated linoleic acid and dietary antibiotics as growth promotants in weanling pigs. Journal of Animal Science. 2001;79:2542-9.

4. Seljelid R, Rasmussen LT, Larm O, Hoffman J. The protective effect of beta 1-3D-glucan-derivatized plastic beads against Escherichia coli infection in mice. Scand J Immunol. 1987;25:55-60.

5. Muchmore AV, Sathyamoorthy N, Decker J, Sherblom AP. Evidence that specific high-mannose oligosaccharides can directly inhibit antigen-driven T-cell responses. J Leukoc Biol. 1990;48:457-64.

6. Podzorski RP, Gray GR, Nelson RD. Different effects of native Candida albicans mannan and mannan-derived oligosaccharides on antigen-stimulated lymphoproliferation in vitro. J Immunol. 1990;144:707-16.

7. Davis ME, Brown DC, Maxwell CV, Johnson ZB, Kegley EB, Dvorak RA. Effect of phosphorylated mannans and pharmacological additions of zinc oxide on growth and immunocompetence of weanling pigs. J Anim Sci. 2004;82:581-7.

8. Li J, Xing J, Li D, Wang X, Zhao L, Lv S, et al. Effects of beta-glucan extracted from Saccharomyces cerevisiae on humoral and cellular immunity in weaned piglets. Archives of Animal Nutrition. 2005;59:303-12.

9. Li J, Li DF, Xing JJ, Cheng ZB, Lai CH. Effects of beta-glucan extracted from Saccharomyces cerevisiae on growth performance, and immunological and somatotropic responses of pigs challenged with Escherichia coli lipopolysaccharide. Journal of Animal Science. 2006;84:2374-81.

10. Davis ME, Maxwell CV, Erf GF, Brown DC, Wistuba TJ. Dietary supplementation with phosphorylated mannans improves growth response and modulates immune function of weanling pigs. Journal of Animal Science. 2004;82:1882-91. 
11. Lee T-I, Kao Y-H, Tsai W-C, Chung C-C, Chen Y-C, Chen Y-J. HDAC Inhibition Modulates Cardiac PPARs and Fatty Acid Metabolism in Diabetic Cardiomyopathy. PPAR Res. 2016;2016:5938740.

12. van Heugten E, Funderburke DW, Dorton KL. Growth performance, nutrient digestibility, and fecal microflora in weanling pigs fed live yeast. Journal of Animal Science. 2003;81:1004-12.

13. Jensen GS, Patterson KM, Yoon I. Yeast culture has anti-inflammatory effects and specifically activates NK cells. Comparative Immunology, Microbiology and Infectious Diseases. 2008;31:487500 .

14. Spring P, Wenk C, Dawson KA, Newman KE. The effects of dietary mannaoligosaccharides on cecal parameters and the concentrations of enteric bacteria in the ceca of salmonella-challenged broiler chicks. Poultry Science. 2000;79:205-11.

15. Pluske JR, Hampson DJ, Williams IH. Factors influencing the structure and function of the small intestine in the weaned pig: a review. Livestock Production Science. 1997;51:215-36.

16. Paiva D, Walk C, McElroy A. Dietary calcium, phosphorus, and phytase effects on bird performance, intestinal morphology, mineral digestibility, and bone ash during a natural necrotic enteritis episode. Poultry Science. 2014;93:2752-62.

17. Ballard ST, Hunter JH, Taylor AE. Regulation of tight-junction permeability during nutrient absorption across the intestinal epithelium. Annual Review of Nutrition. 1995;15:35-55.

18. Furuse $M$, Hirase $T$, Itoh $M$, Nagafuchi A, Yonemura $S$, Tsukita $S$, et al. Occludin: a novel integral membrane protein localizing at tight junctions. The Journal of Cell Biology. 1993;123:1777-88.

19. Günther C, Neumann H, Neurath MF, Becker C. Apoptosis, necrosis and necroptosis: cell death regulation in the intestinal epithelium. Gut. 2013;62:1062-71.

20. Kluger MJ. Fever: role of pyrogens and cryogens. Physiological Reviews. 1991;71:93-127.

21. Mazmanian SK, Round JL, Kasper DL. A microbial symbiosis factor prevents intestinal inflammatory disease. Nature. 2008;453:620-5.

22. Maloy KJ, Powrie F. Intestinal homeostasis and its breakdown in inflammatory bowel disease. Nature. 2011;474:298-306.

23. Wannemacher RW. Key role of various individual amino acids in host response to infection. The American Journal of Clinical Nutrition. 1977;30:1269-80.

24. Johnson RW. Inhibition of growth by pro-inflammatory cytokines: an integrated view. Journal of Animal Science. 1997;75:1244-55.

25. Sun Y, Wu C, Ma J, Yang Y, Man X, Wu H, et al. Toll-like receptor 4 promotes angiogenesis in pancreatic cancer via PI3K/AKT signaling. Experimental Cell Research. 2016;347:274-82.

26. Becker CE, O'Neill LAJ. Inflammasomes in inflammatory disorders: the role of TLRs and their interactions with NLRs. Seminars in Immunopathology. 2007;29:239-48.

27. Akira S. TLR signaling. Current Topics in Microbiology and Immunology. 2006;311:1-16.

28. Kontoyiannis D, Kotlyarov A, Carballo E, Alexopoulou L, Blackshear PJ, Gaestel M, et al. Interleukin-10 targets p38 MAPK to modulate ARE-dependent TNF mRNA translation and limit intestinal pathology. 
The EMBO journal. 2001;20:3760-70.

29. Duan X, Tian G, Chen D, Huang L, Zhang D, Zheng P, et al. Mannan oligosaccharide supplementation in diets of sow and (or) their offspring improved immunity and regulated intestinal bacteria in piglet1. Journal of Animal Science. 2019;97:4548-56.

30. Sun Y, Shi X, Zheng X, Nie S, Xu X. Inhibition of dextran sodium sulfate-induced colitis in mice by baker's yeast polysaccharides. Carbohydrate Polymers. 2019;207:371-81.

\section{Figures}
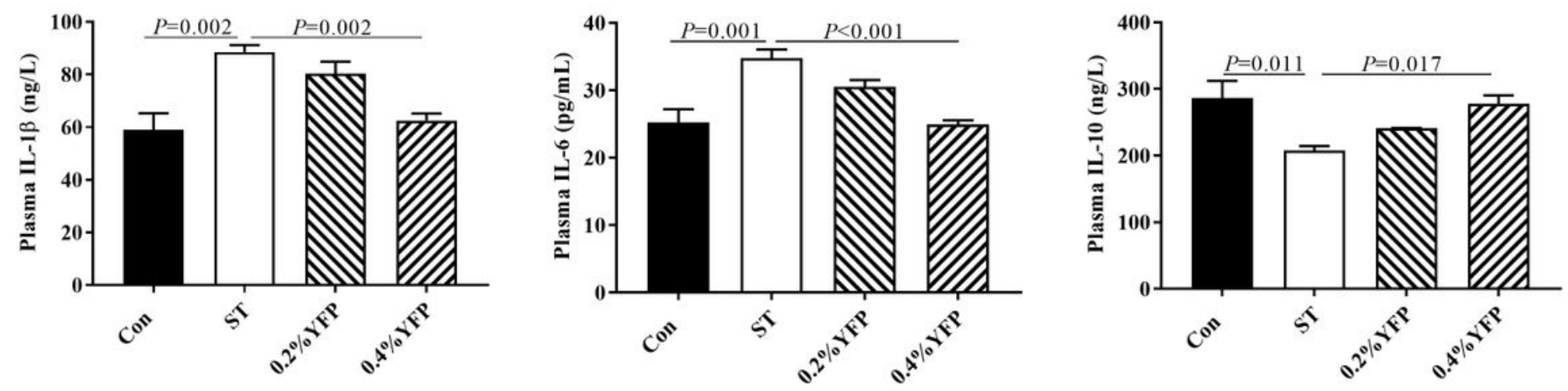

Figure 1

Effect of YFP supplementation on concentration of plasma inflammatory cytokines after Salmonella typhimurium challenge in piglets. The values are presented as mean \pm SEM. 
A

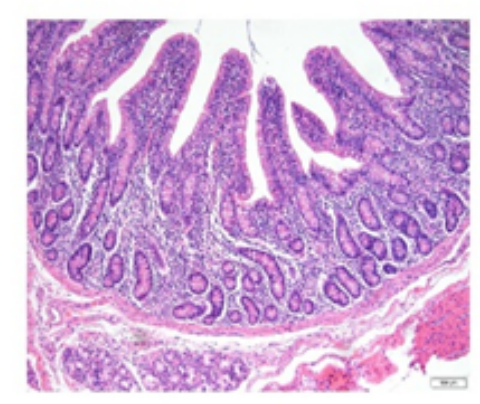

Con

B

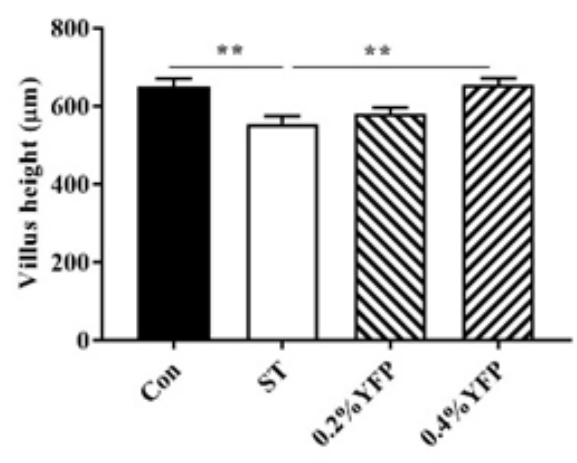

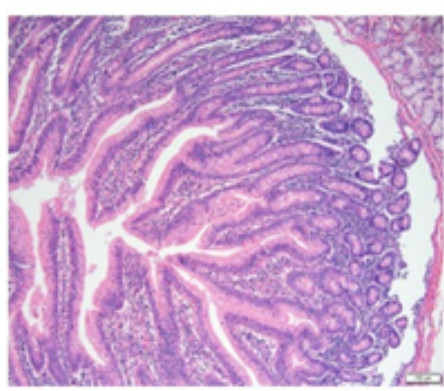

ST

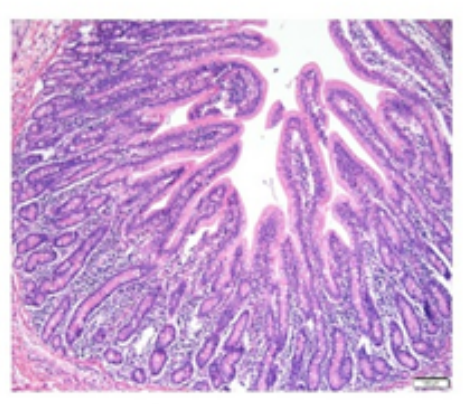

$0.2 \%$ YFP

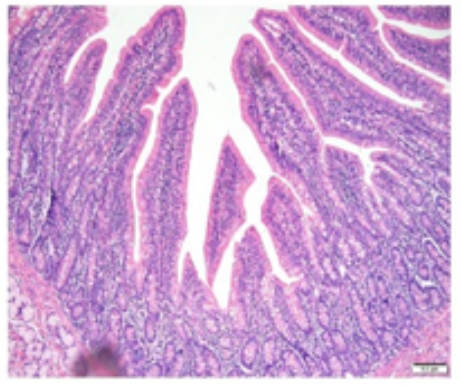

$0.4 \%$ YFP
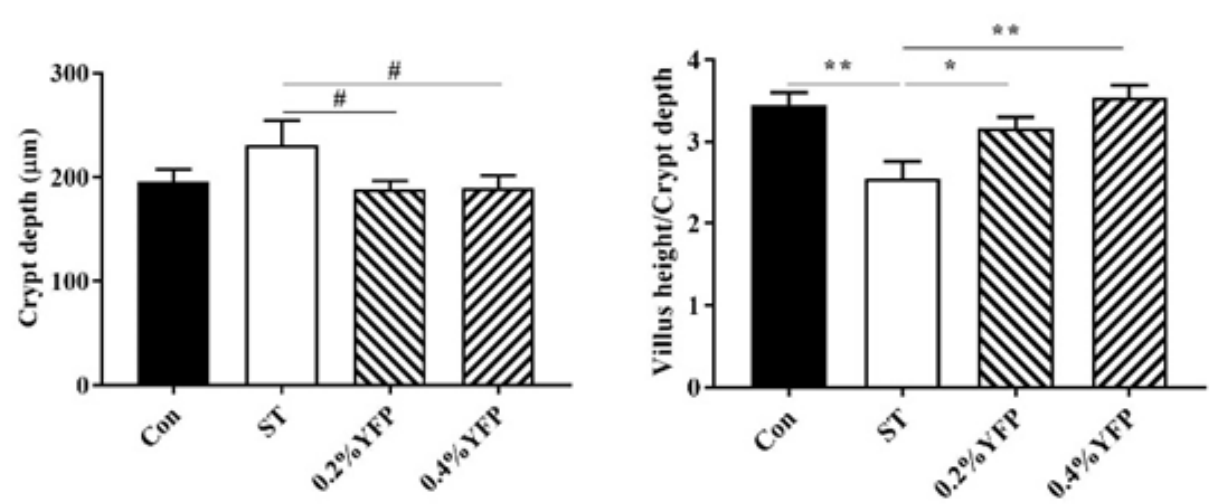

Figure 2

Effect of YFP supplementation on duodenal morphology after Salmonella typhimurium challenge in piglets. The values are presented as mean \pm SEM. ${ }^{*} P<0.05, * * P<0.01, \# P<0.1$ 


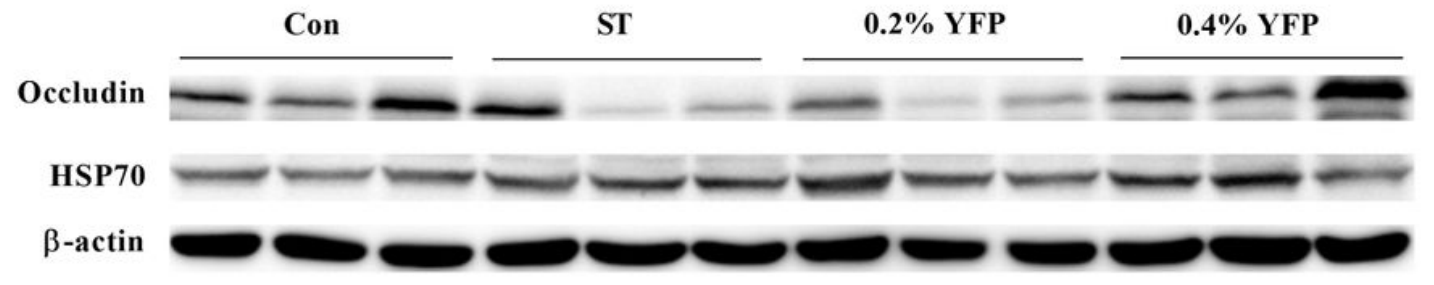

$\mathbf{A}$

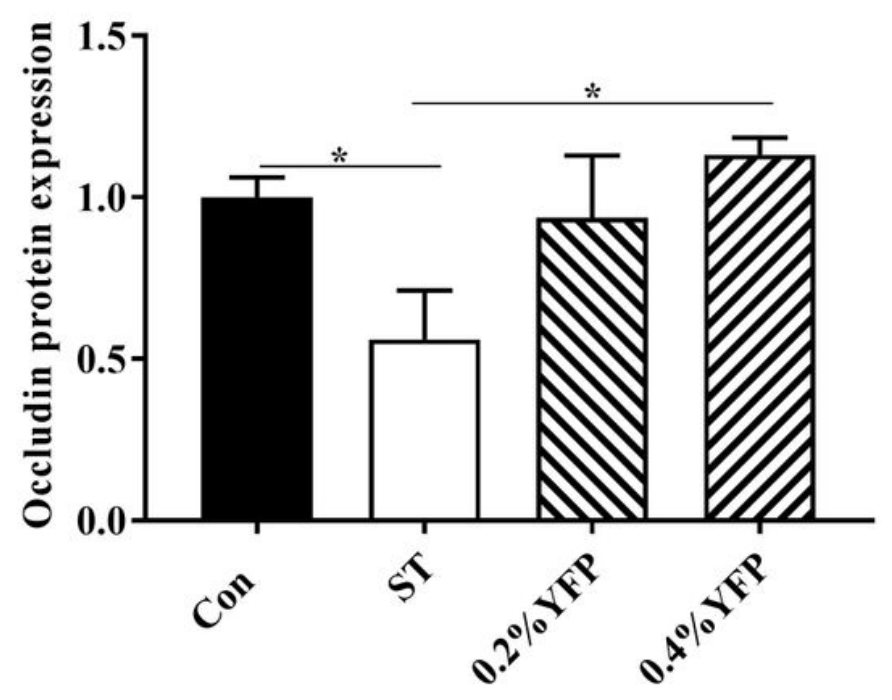

B

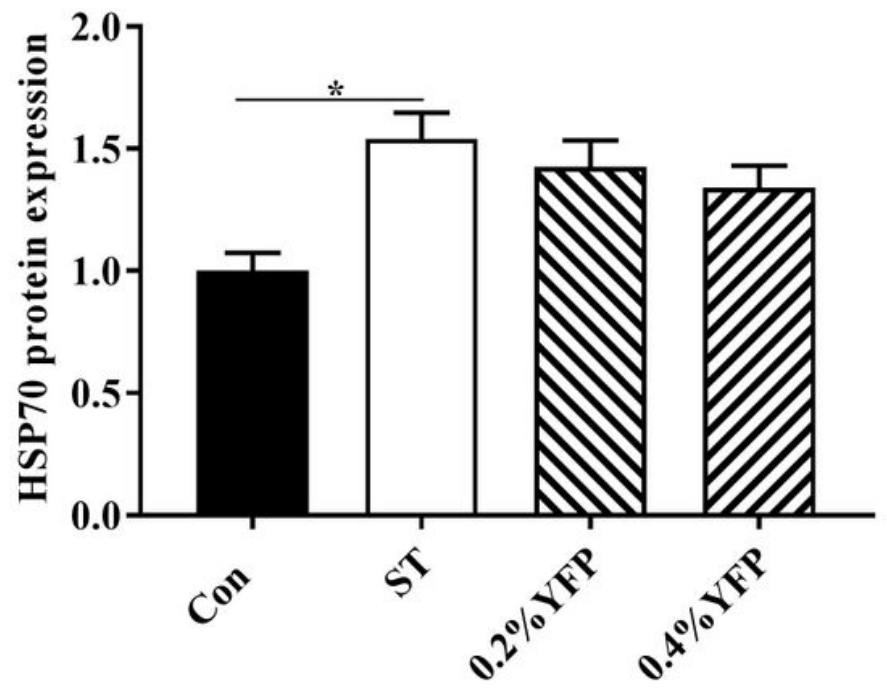

Figure 3

Effect of YFP supplementation on occludin and heat shock protein 70 (HSP 70) protein expression in duodenum after Salmonella typhimurium challenge in piglets. $\beta$-actin were used for normalization for relative occludin and HSP70 expression. The values are presented as mean $\pm \mathrm{SEM}$. ${ }^{*} \mathrm{P}<0.05$ 
A
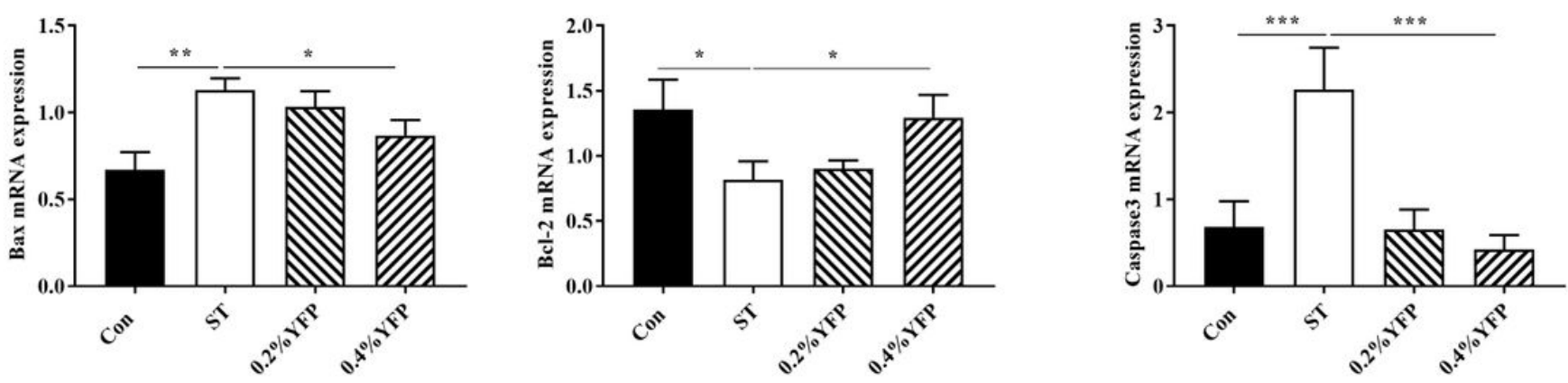

B

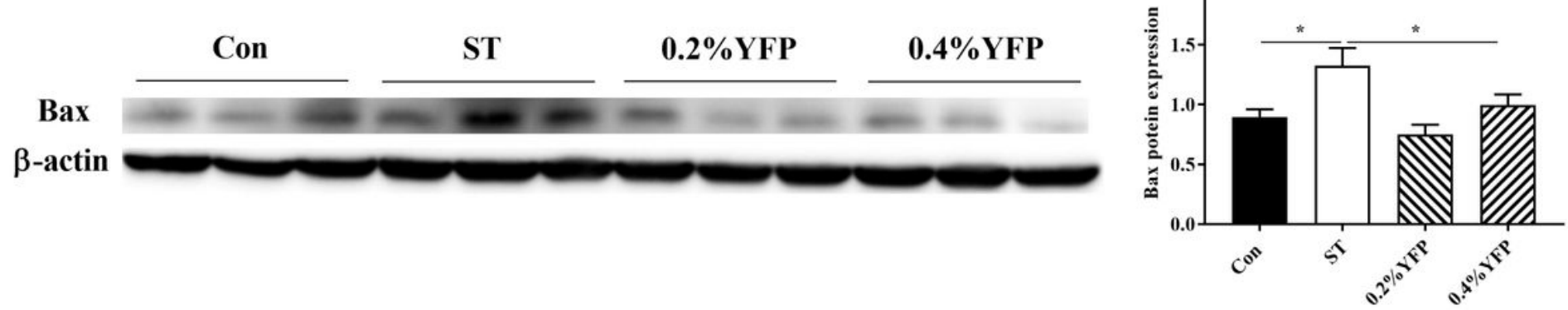

Figure 4

Effect of YFP supplementation on mRNA expression of key molecules of apoptosis signaling after Salmonella typhimurium challenge in piglets. The values are presented as mean $\pm S E M$. ${ }^{*} P<0.05, * \star P<$ 0.01 
A
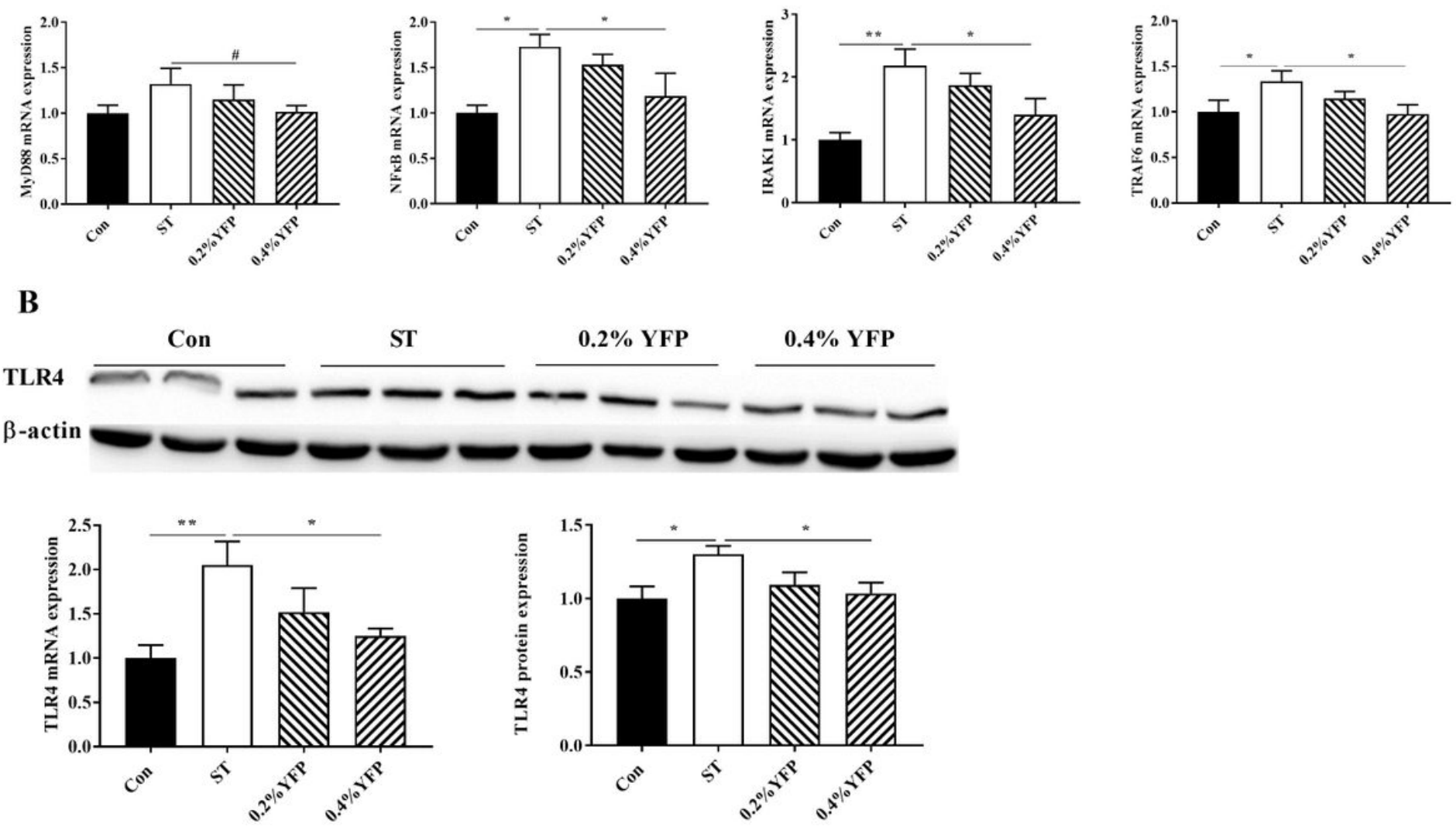

Figure 5

Effect of YFP supplementation on mRNA and protein expression of inflammation-related signaling molecules after Salmonella typhimurium challenge in piglets. $\beta$-actin were used for normalization for relative TLR4 expression. The values are presented as mean \pm SEM. ${ }^{*} \mathrm{P}<0.05,{ }^{\star *} \mathrm{P}<0.01$ 

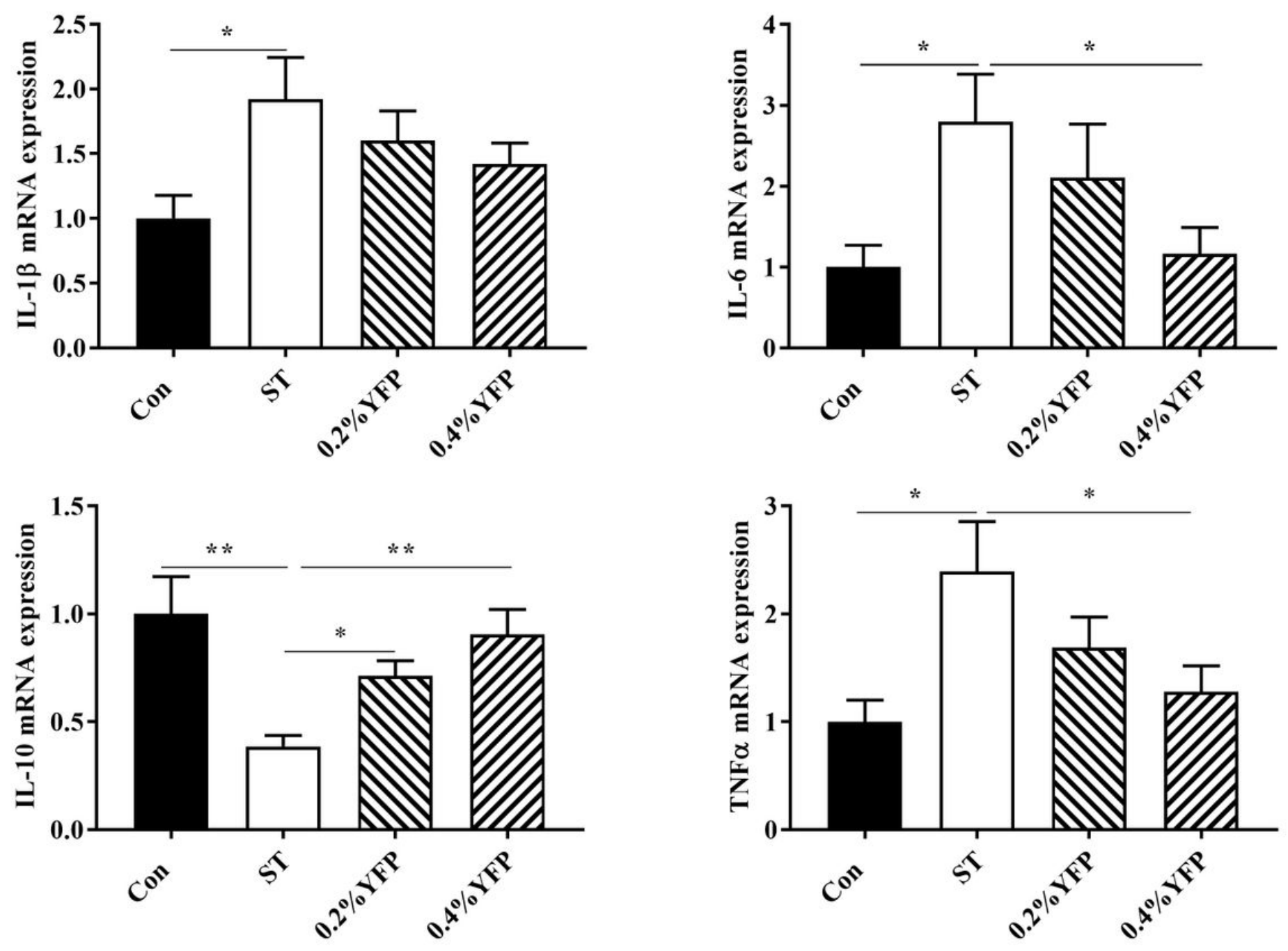

Figure 6

Effect of YFP supplementation on mRNA expression of inflammation cytokines in duodenum after Salmonella typhimurium challenge in piglets. The values are presented as mean $\pm S E M$. ${ }^{*} P<0.05, * \star P<$ 0.01 

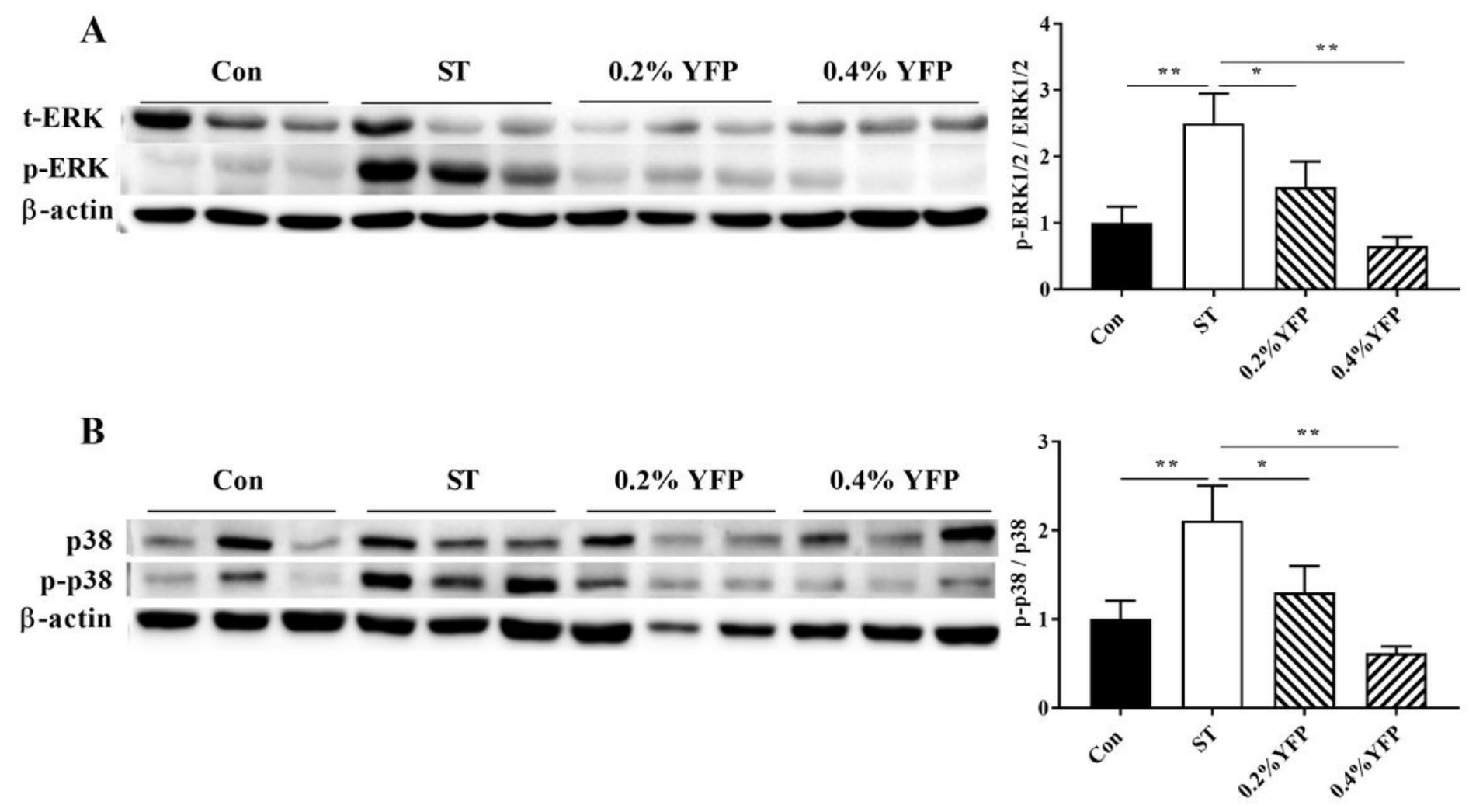

Figure 7

Effect of YFP supplementation on duodenal extracellular signal-related kinase 1/2 (ERK1/2) and p38 phosphorylation levels after Salmonella typhimurium challenge in piglets. Phosphorylated forms of ERK1/2 and p38 were normalized to the total amount of each protein. The values are presented as mean \pm SEM. $* P<0.05, * * P<0.01$ 